\section{The intriguing new possibilities of the microbiological methodology and molecular diagnostics for water quality analysis}

Before the emergence of COVID-19 (SARS-CoV2), who would have thought that a pandemic would turn out to be a broad publicity vehicle for the application of molecular biological and biochemical methods? Technical terms such as PCR and antigen testing, even nucleic acid sequencing, are now part of the standard repertoire of the "normal" earthling. And it has become clear to almost everyone by now that without the use of these powerful methods, crisis management would resemble flying blind. For the time being, the widespread use of these methods is unthinkable without them.

A similar "acceptance gain" with regard to an analytical-diagnostic methodology must have taken place about 150 years ago, albeit, without the help of social media and television, remaining limited to the narrower professional community. Nobel Prize winner Robert Koch \& Co were the first to identify pathogens, rather than the postulated evil vapors (miasmas), as the cause of disease outbreaks, and furthermore to trace sources of pollution and infection in the environment. This so-called "golden age" of microbiology laid the foundation for cultivation-based standard methods for the detection of microbiological quality indicators and pathogens: a methodological quantum leap. Detection-for example, of the fecal indicator bacterium Escherichia coli (E. coli)-has since been used in qualityassured laboratories based on colony formation by cell division on culture media for microbiological hygiene testing of water.

The need for complementary methods of extension Cultivation-based standard methods are essential in the accredited monitoring of microbiological-hygienic water quality (e.g. drinking and human use, recreation/swimming, irrigation). They are a fundamental element in modern water resource quality management for public health. Without them, daily life in this form would be unimaginable. Nevertheless, many broader questions and problems in the water field cannot be addressed or solved adequately using cultivation-based standard methods. This need for methodological supplements is reinforced by the increasing requirements for quality specifications, such as risk-based analysis targets or the need for clarification of the cause or origin of microbiological contamination. The possibility to open the microbiological "black-box" is therefore increasingly demanded in many cases in the course of a pro-active and sustainable resource and quality management.

The new possibilities of biotechnology \& life sciences The rapid developments in the field of life sciences, biotechnology and information technology as well as automation technology have created impressive possibilities for microbiological and molecular biological diagnostics in the last decades. With the help of modern molecular biological and biochemical methods, microorganisms and viruses, for example, can be broken down into their "building blocks" (e.g. bases of nucleic acids or amino acids of proteins) in order to derive information on identity, origin, and often also relevance or activity. The generated data sets can then be archived using bioinformatics analysis tools, compared with reference databases and ultimately evaluated (e.g. identification of unknown viral or bacteriological isolates). Many of the questions that were completely unsolvable in the microbiological examination of water a few years ago have become accessible for analysis with these modern methods. In this context, it is important to note that these new methods can in no way replace the legally prescribed examination standards, but can complement them if necessary.

The ÖWAV working committee microbiology and water This working committee of the Quality and Hygiene Division has set itself the goal of continuously discussing the new possibilities of microbiological methodology and molecular biological diagnostics for solving current problems in the water sector and to make the essential developments and information available at the expert level-as comprehensibly as possible. The methodical-thematic range is broadly based in the sense of the ÖWAV

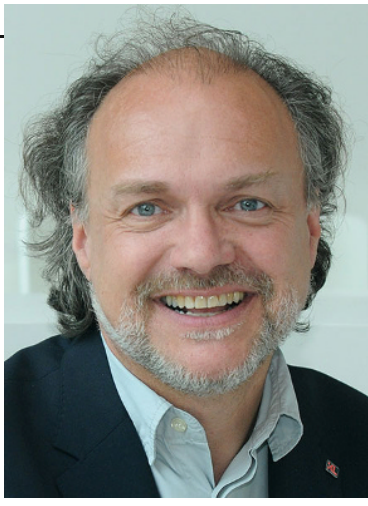

(c) KL/Andrea Reischer

Univ.-Prof. PD Dr. Andreas Farnleitner

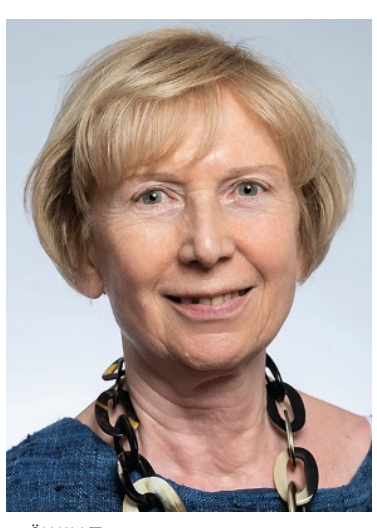

(c) ÖWAV/Titzer

Dr. Franziska Zibuschka 
and includes microbiological-hygienic questions of the human use of water resources (as already mentioned above), biotechnological-analytical aspects in the water sector (e.g. microbiome analyses in biological wastewater treatment, biostability investigations in water supply), as well as, not to forget, microbial-ecological aspects of our waters and water resources. From a methodological point of view, the field of wastewater-based epidemiology of pathogens has also gained new importance and media presence, as demonstrated by the current SARS-CoV-2 surveillance. The activities of the ÖWAV working committee are not limited to a few water types or compartments, but are discussed as comprehensively as possible (e.g. surface water, groundwater, drinking water, wastewater).

Several seminars have already been held on the committee's activities in recent years. At the 2017 seminar, the ÖWAV working aid 52 "Microbiology and Water, Part 1-Overview of Methodological Options for Analysis" was also presented (ÖWAV 2017). This work aid attempts to provide a brief and comprehensible overview for the practitioner in the water sector on the currently available analytical methods. In addition to the cultivation-based standard methods, the variety of supplementary methods is presented and explained ("from DNA/RNA diagnostics to on-line measurement technology"). In 2022, another seminar of this committee will now take place again, where the supplementary ÖWAV work aid "Microbiology and Water, Part 2-Case Studies to Illustrate the New Diagnostic-Analytical Possibilities" (ÖWAV 2022) will be presented. The focus in this work aid will be on demonstrating the possible solutions to microbiological problems in water using the new complementary microbiological methodology and molecular diagnostics.

Aim and content of the present ÖWAW volume 11-12/21 The thematic focus "The New Possibilities of Microbiological Diagnostics for the Analysis of Water Quality" of the Austrian Water and Waste Management can be seen in a way as the official and visible starting signal for the planned activities 2022 of the ÖWAV Working Committee Microbiology and Water. A number of original contributions could be edited, which in sum should give a first impressive and concise insight into the new possibilities of microbiological methodology and molecular diagnostics to address current or unsolved microbiological problems of the water sector. Many thanks to all authors for their efforts leading to the successful realization of this thematic focus. In contrast to the workbook "Microbiology and Water, Part 2-Case Studies to Illustrate New Diagnostic-Analytical Possibilities" planned for 2022 (ÖWAV 2022), the original papers in this ÖWAW 11-12/21 issue are in English. This is to reach the nonGerman speaking (international) ÖWAW readership.

The monitoring and also assessment of the ecological quality status of groundwater is a hot topic (Griebler et al. 2014). Different assessment tools have been developed and are waiting for their application and validation. One of them is the so-called D-A-C index. This evaluates the microbiological-ecological quality of groundwater based on the total bacterial cell count (prokaryotic cells), the measurement of microbial activity, and the qualitative characterization of dissolved organic carbon (DOC). In the paper by Retter et al. the various applications of the D-A-(C) index are presented based on a recently collected data set from the River Mur Valley, Austria. First, the extension of the D-A-(C) index is demonstrated by including measurements of DOC quality obtained by fluorescence spectroscopy as additional variable to complement the analysis. In addition, it is shown how defining a reference condition for "good" microbiological ecological status can improve the analysis and allow more sensitive detection of changes or impacts to groundwater ecosystems.

In addition to recording the ecological quality status of surface and ground waters, the question of the extent and origin of microbiological fecal contamination of water and water resources has been of central importance since the beginning of the field of microbiology, due to its major health significance. Standardized cultivation-based detection of fecal indicators, such as $E$. coli or intestinal enterococci, provides reliable information on the quantity of contamination, but no information on the origin, since it occurs in both animal and human sources. In the paper by Steinbacher et al. the applicability of so-called host-associated genetic fecal markers (PCR-based DNA quantification) for source determination of fecal contamination-also referred to as microbial source tracking (MST)-is tested comprehensively for the first time for water resources in Lower Austria and their potential 


\section{Editorial}

sources of input (i.e., municipal wastewater disposal, livestock, wildlife). Furthermore, the combined use with cultivation-based fecal indicators is demonstrated.

In connection with the PCR-based detection of genetic targets in water, a contribution to the detection of SARS-CoV-2 RNA in municipal raw sewage was also planned in the course of the Austrian activities for sewage-based COVID-19 surveillance. Unfortunately, this contribution could not be realized in time. It is hoped that contributions on this topic can be realized in upcoming ÖWAW issues as well as at the lecture event planned for next year.

The combination of the fields of microbiology and hydrology is of particular importance for providing solutions to problems in water management. In particular, linking microbiological measurement data (e.g. concentrations of indicators and pathogens) with mathematical hydraulic transport and dispersion modeling in water resources can derive fundamental information for the technical implementation and dimensioning of management concepts (e.g., design of protection zones in drinking water production or treatment measures (Demeter et al. 2021)). In the paper by Derx et al., the transport of fecal indicators (E. coli and spores of Clostridium perfringens) into groundwater is modeled for an Austrian section of the Danube including an oxbow lake. For this purpose, a 3-D unsaturated-saturated groundwater model coupled with a 2-D hydrodynamic flow model is used. The methodological approach presented shows how hydraulic transport modeling and microbiological analyses can be combined to support decision making in planning activities.

In addition to threats from fecal contamination, the microbiological quality of groundwater can also be affected by the growth of the natural water microbiome, for example, due to nutrient inputs. This can lead to high concentrations of opportunistic pathogens, impaired odor and taste, or biocorrosion when used in water supply or industrial uses. The occurrence of such phenomena indicates "biological instability", which may lead to quality problems in the use of the water resource (e.g. distribution, storage). It should be explicitly pointed out that the natural water microbiome is part of the very essence of groundwater. Groundwater and drinking water are not sterile, and the water microbiome from nutrient-poor groundwater has no negative health or technical relevance in the water supply. In the paper of Kirschner et al. a new integrative, two-step approach consisting of in-situ and predictive methods is applied to comprehensively assess the biological stability of groundwater in the course of a bank filtration system of the Danube River. Traditional cultivation-based as well as selected cultivation-independent methods (total cell count by microscopy and flow cytometry (FZ), leucine isotope incorporation, 16S-rRNA-gene amplicon sequencing) are comparatively applied along the infiltration pathway from the river to the groundwater well to visualize different aspects in the investigation of biostability.

As the final paper, the contribution by Schönher et al. provides focused insight into the methodology and applicability of FZ for analyzing the microbiological characteristics of groundwater as a drinking water resource and in the course of water distribution. FZ can provide high-resolution information at the single-cell level, including total cell count, size measurement, nucleic acid content, and viability and viability of captured bacterial populations (prokaryotic cells). Several case studies are presented demonstrating the wide range of different objectives and capabilities, including results on flow cytometric characterization of selected Austrian drinking water resources and advanced data analysis at FZ.

The interview with DI Wolfgang Vogl brought after the scientific articles briefly informs about the national and international activities of an Austrian company (VWMS Gmbh, Zwerndorf) in the field of microbiological-biochemical on-line monitoring. Automation and on-line measurement technology for "near real-time analysis" of microbiological quality characteristics is of increasing international importance. It is good to see that an Austrian company is doing pioneering work here in the development of measurement technologies and equipment and its application. A short overview on the history of the company as well as the applied measurement principle and technical background information is given. 
Concluding remarks The use of new complementary microbiological, biochemical and molecular biological analytical methods in the water sector has just begun. A recent methodological quantum leap, such as in the days of Robert Koch, seems to be underway. To ensure adequate use of these new possibilities, close cooperation between water management practice and university research is essential. One thing seems to be essential: Every methodology has its possibilities and limitations. There are no universally applicable research methods, only suitable "tools" for well-defined questions. Experts are required to provide the appropriate information.

\section{References}

Demeter, K., Derx, J., Komma, J., Parajka, J., Schijven, J., Sommer, R., Cervero-Arago, S., Lindner, G., ZoufalHruza, C.M., Linke1, R., Savio, D., Ixenmaier, S., Kirschner, A.K.T., Kromp, H., Blaschke, A.P. \& Farnleitner A.H. (2021): Modelling the interplay of future changes and wastewater management measures on the microbiological river water quality considering safe drinking water production, Science of the Total Environment 768:144278.

Griebler, C., Malard, F. \& Lefébure, T. (2014): Current developments in groundwater ecology-from biodiversity to ecosystem function and services. Current Opinion in Biotechnology 27:159-167.

ÖWAV (2017): Mikrobiologie und Wasser. Teil 1: Übersicht zu den methodischen Möglichkeiten der Analyse. ÖWAVArbeitsbehelf 52, Wien 2017

ÖWAV (2022): Mikrobiologie und Wasser. Teil 2: Fallstudien zur Illustration der neuen diagnostisch-analytischen Möglichkeiten. ÖWAV-Arbeitsbehelf, Wien 2022 (in prep.)

\section{Univ.-Prof. PD Dr. A. Farnleit- \\ ner, MSc.Tox.}

Interuniversity Cooperation Centre

Water \& Health (ICC Water \& Health),

www.waterandhealth.at,

Vienna - Krems, Austria

Division Water Quality and Health,

Karl Landsteiner University for Health

Sciences,

Dr.-Karl-Dorrek-Straße 30,

3500 Krems an der Donau, Austria

Institute of Chemical, Environmental

and Biological Engineering, 166/5/3,

TU Wien,

Gumpendorferstraße 1A,

1060 Wien, Austria

andreas.farnleitner@tuwien.ac.at

\section{Dr. F. Zibuschka (ه)}

Innocentiagasse 9 ,

1130 Wien, Austria

franziska.z@netm.at 